\title{
Retarded Harrier Maneuver as a New and Efficient Approach for Fixed-Wing Aircraft to Achieve S/VTOL
}

\author{
Chung-Kiak Poh, Chung-How Poh \\ Aero-Persistence Research, Penang, Malaysia \\ Email:kiak@aero-persistence.com
}

How to cite this paper: Poh, C.-K. and Poh, C.-H. (2021) Retarded Harrier Maneuver as a New and Efficient Approach for Fixed-Wing Aircraft to Achieve S/VTOL. Advances in Aerospace Science and Technology, 6, 81-92.

https://doi.org/10.4236/aast.2021.62006

Received: March 1, 2021

Accepted: May 24, 2021

Published: May 27, 2021

Copyright (c) 2021 by author(s) and Scientific Research Publishing Inc. This work is licensed under the Creative Commons Attribution International License (CC BY 4.0).

http://creativecommons.org/licenses/by/4.0/

\begin{abstract}
Modern day VTOL fixed-wing aircraft based on quadplane design is relatively simple and reliable due to lack of complex mechanical components compared to tilt-wings or tilt-rotors in the pre-80's era. Radio-controlled aerobatic airplanes have thrust-to-weight ratio of greater than unity and are capable of performing a range of impressive maneuvers including the so-called harrier maneuver. We hereby present a new maneuver known as the retarded harrier that is applicable to un/manned fixed-wing aircraft for achieving VTOL flight with a better forward flight performance than a quadplane in terms of weight, speed and esthetics. An airplane with tandem roto-stabilizers is also presented as an efficient airframe to achieve VTOL via retarded harrier maneuver, and detailed analysis is given for hovering at $45^{\circ}$ and $60^{\circ}$ and comparison is made against the widely adopted quadplane. This work also includes experimental demonstration of retarded harrier maneuver using a small remotely pilot airplane of wingspan $650 \mathrm{~mm}$.
\end{abstract}

\section{Keywords}

Fixed-Wing Aircraft, Roto-Stabilizer, Vertical Takeoff and Landing, Short Takeoff, Harrier Maneuver, Distributed VTOL System (DVS), Urban Air Mobility (UAM)

\section{Introduction}

\subsection{Fixed-Wing Aircraft: Quest for VTOL}

One of the earliest concepts of fixed-wing aircraft with vertical takeoff and landing (VTOL) capability was the P.1003/1 by Weserflug in 1938 [1] [2]. By 1990's, there were several tiltrotors and tilt-wings with VTOL capability despite the 
mechanical complexities associated with rotatable engine nacelles or wings [1] [3] [4]. The quadplane is a relatively newcomer in the development of VTOL airplanes. A quadplane is built upon an airplane with at least one horizontal propulsor and at least four rotors or propellers for vertical flight [5]. When a quadplane is in forward flight, its powerful vertical propulsors are inactive and having zero contribution to forward propulsion. It is not uncommon to encounter airframes used to realize electric VTOL or eVTOL to be largely based on the quadplane design [6] [7] [8].

\subsection{Aerobatic Airplanes and 3D Maneuvers}

Modern aerobatic fixed-wing aircraft, whether full-size or in the form of a radio controlled (RC) model, are designed to operate even under fully stalled conditions [9] [10] [11] [12] [13]. These fixed-wing aircraft can fly in trim at high angles of attack of $45^{\circ}$ or more with air speed below the stall speed $\left(V_{\mathrm{s}}\right)$-a maneuver known as the "harrier" and it is one of the popular "3D aerobatic maneuvers" within the RC flight community [13] [14]. Hallmarks of unlimited aerobatic airplanes are their relatively large control surfaces and a thrust-to-weight ratio that exceeds unity [9] [10]. Large control surfaces with large deflections in the presence of strong propeller wash give adequate authority even when the airspeed of the aircraft is much lower than the stall speed $V_{s}[14]$.

\subsection{Tandem Roto-Stabilizers}

In our previous work, we introduced the concept of roto-stabilizer, both in mono [15] and tandem configurations [16]. Mono roto-stabilizer is a viable substitution for conventional horizontal stabilizer [15] [16]. The tandem roto-stabilizers provides rotor-borne lift in addition to exerting pitching moment and it was specifically developed to achieve hyper-short takeoff and landing (hyper-STOL) or VTOL via a maneuver known as "retarded harrier"-a maneuver similar to the harrier except for the presence of an opposing aerodynamic force that opposes any forward thrust in the horizontal direction. Figure 1 shows a photorealistic

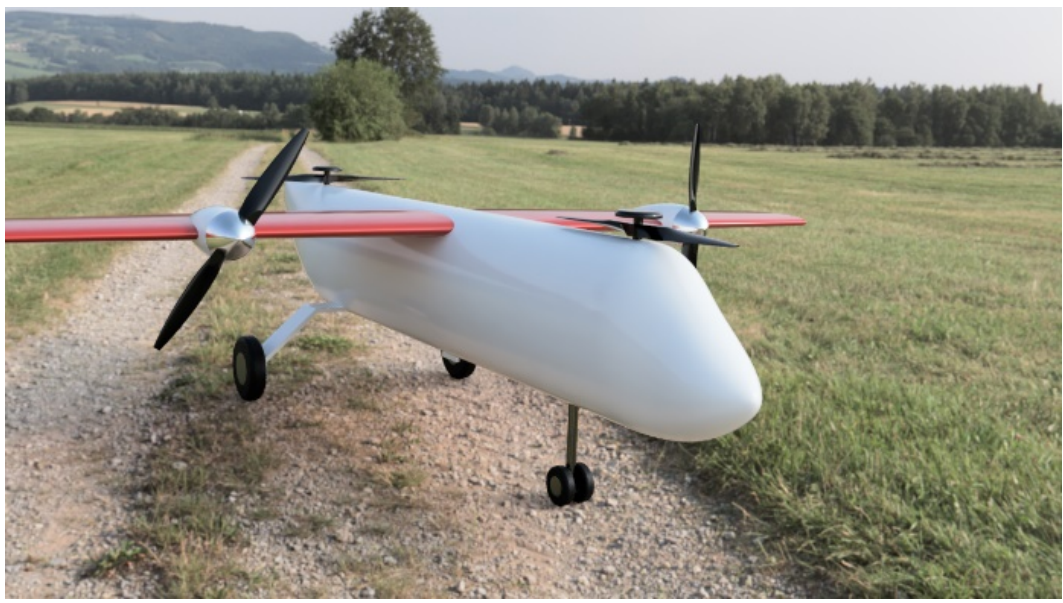

Figure 1. Concept of roto-stabilizers for achieving VTOL via retarded harrier maneuver. 
render of an airplane with tandem roto-stabilizers. The rendering was done in Autodesk Fusion $360^{\circ}$. The tandem roto-stabilizers are counter-rotating to each other for torque cancelation and the same applies to the primary horizontal propulsion units mounted on the wings [16]. The roto-stabilizers are located at approximately equal distance from the C.G. of the airplane, i.e., one towards the front and the other one towards the rear of the airplane [16].

The tandem roto-stabilizers provide partial lift during takeoff and landing, helping to reduce forward airspeed thereby making the ground roll distance extremely short resulting in hyper-STOL. Each of the tandem roto-stabilizers can be regarded as a vertical propulsor. An airplane with tandem roto-stabilizers (ATRS) is a potential candidate for urban air mobility (UAM) applications [16].

A force diagram for the airplane with tandem roto-stabilizers is illustrated in Figure 2. The airplane has an all-up-weight (AUW) of $W_{\Sigma}$. Each horizontal propulsor produces a thrust $T_{\text {hoz }}$, and each vertical propulsor produces a thrust $T_{\text {vert }}$. At certain conditions, such as when the pitch angle, $\rho$ is $45^{\circ}$, and when all of the propulsors generate equal amount of thrust (i.e., $T_{\text {vert }}=T_{\text {hoz }}$ ), hovering is achieved thus making VTOL flights possible without the need for complex rotatable mechanisms.

During a stationary hover, the expression for the horizontal components [16] is

$$
T_{\text {hoz }} \cos (\rho)=T_{\text {vert }} \sin (\rho)
$$

and the expression for the vertical components is

$$
T_{\text {hoz }} \sin (\rho)+T_{\text {vert }} \cos (\rho)=0.5 \times W_{\Sigma}
$$

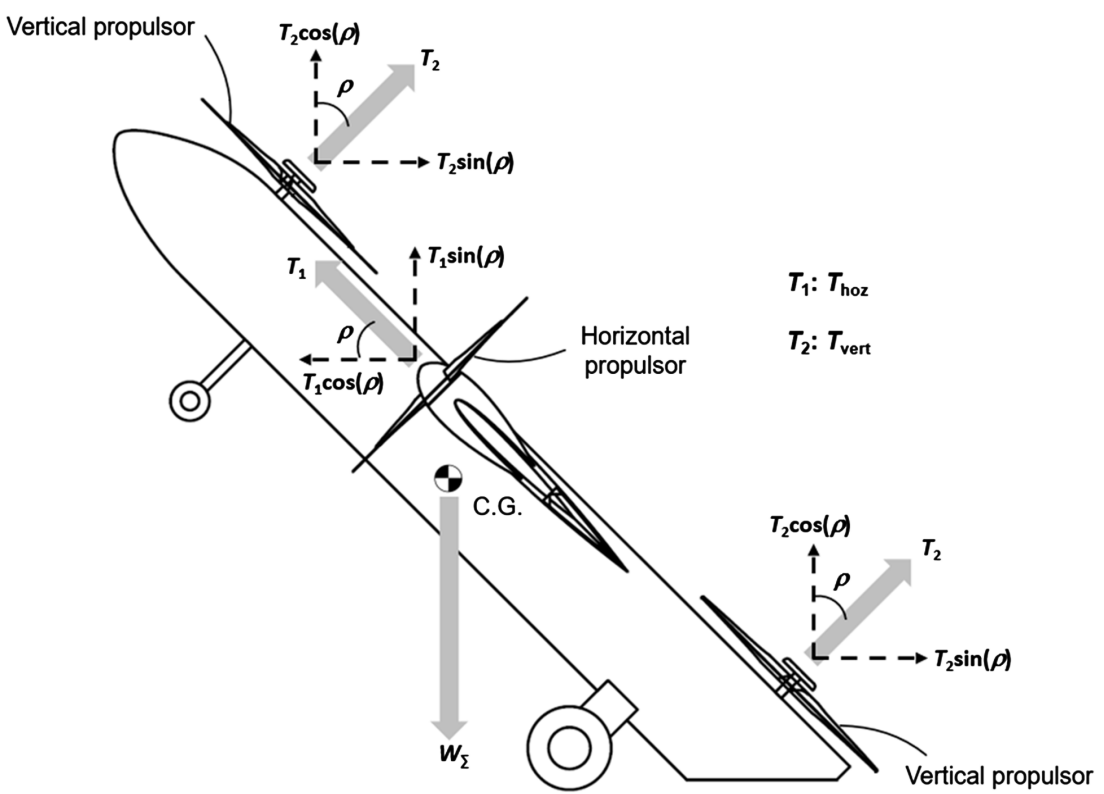

Ground

Figure 2. Force diagram for the aircraft with tandem roto-stabilizers. 


\section{Results and Discussion}

\subsection{Analysis}

Based on Equations (1) and (2), the magnitude of $T_{\text {hoz }}$ and $T_{\text {vert }}$ required to sustain a hover can be established. A plot of $T_{\text {hoz }}$ and $T_{\text {vert }}$ as a function of pitch angle $\rho$ in the range of $0^{\circ}$ to $60^{\circ}$ is as shown in Figure 3. The thrust generated by each propulsor is $0.3536 W_{\Sigma}$ when the aircraft is hovering with a pitch angle $\rho$ of $45^{\circ}$. This means that for a given AUW, each of the four propulsors of the aircraft needs to generate some $10 \%$ more thrust than that of a quadplane in order to sustain a hover. However, hovering and VTOL are often transitory in most applications including urban air mobility and therefore such increase is not expected to decrease the overall flight time significantly, and this is especially so for those involving long haul flights. Likewise from Figure 3, when the airplane is hovering with a pitch angle $\rho$ of $60^{\circ}, T_{\text {hoz }}$ is $0.4330 W_{\Sigma}$ and $T_{\text {vert }}$ is $0.25 W_{\Sigma}$. For ease of reference, the airplanes with tandem roto-stabilizers hovering with pitch angle $\rho$ of $45^{\circ}$ and $60^{\circ}$ are referred to as ATRS-45, and ATRS-60, respectively.

A typical quadplane and a fixed-wing aircraft with tandem roto-stabilizers are illustrated in Figure 4(a) and Figure 4(b), respectively. Thrust magnitudes

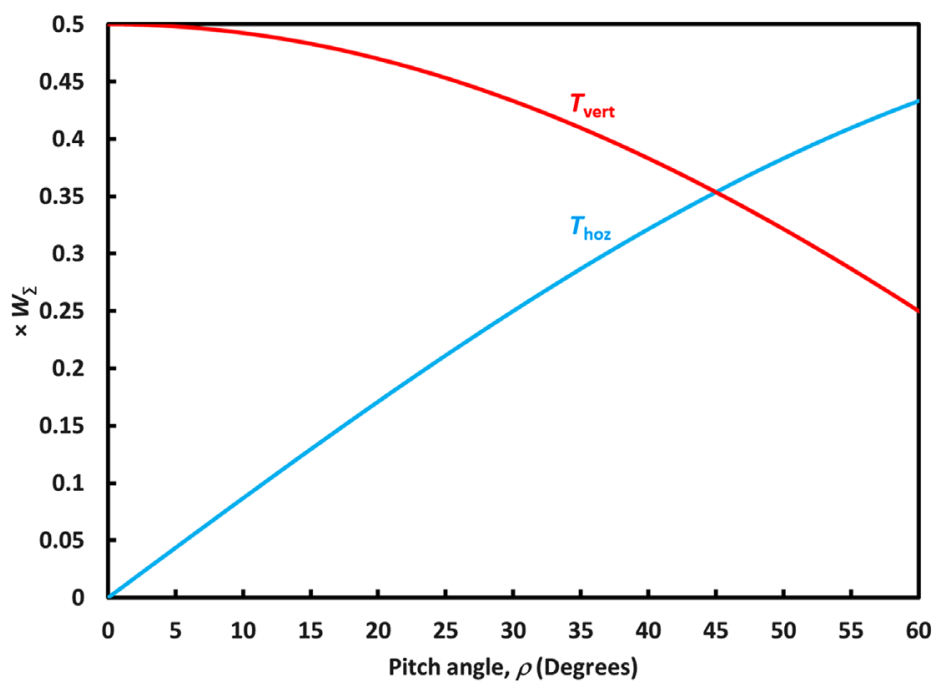

Figure 3. Values of $T_{\text {hoz }}$ and $T_{\text {vert }}$ required to achieve hover for a given pitch angle, $\rho$.

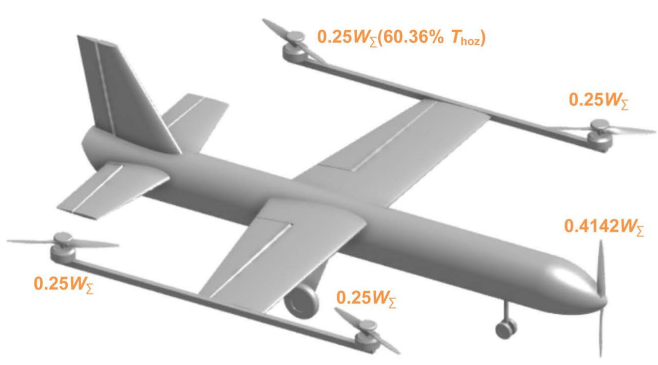

(a)

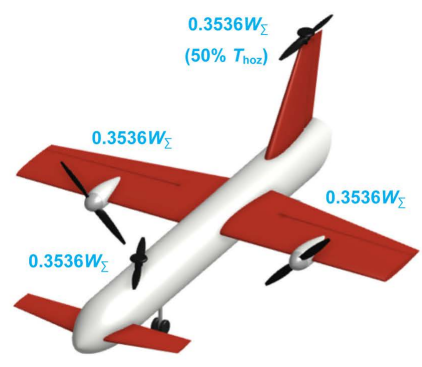

(b)

Figure 4. Types of fixed-wing VTOL aircraft being considered in the analysis: (a) quadplane, and (b) airplane with tandem roto-stabilizers. 
shown in Figure 4(b) are for ATRS-45 and they were derived from the plot in Figure 3. As mentioned, during forward flight, all of the quadplane's four vertical propulsors are neither active nor contributing to forward thrust, and therefore it was appropriate to consider the weight of the vertical propulsors as a "weight penalty" that contributes to degradation in forward flight performance. Based on the same argument, the ATRS would have two vertical propulsors that are inactive during forward flight.

In order to obtain a "weight penalty" model for the two aircraft types and to make comparison possible, both aircraft were assigned the same AUW of $W_{\Sigma}$ and both aircraft were given the same amount of total propulsion power (horizontal, plus vertical). Furthermore, it was assumed that the propulsors of both aircraft were brushless electric motors of $100 \%$ efficiency and all of the motors having identical gravimetric power density, $\gamma$.

Hence, we can write

$$
\gamma=\frac{P}{m}
$$

where $P$ is the power of the brushless motor, and $m$ is the mass of the individual motor.

Equation (3) also implies that if two aircraft have the same total power, they would have the same total propulsor weight. We defined "weight penalty", $W_{\text {pe- }}$ nalty to be the weight ratio of the vertical to the horizontal propulsors

$$
W_{\text {penalty }}=\frac{W_{\text {vert }}}{W_{\text {hoz }}}
$$

The premise for the definition of $W_{\text {penalty }}$ was based on the notion that it is the forward thrusts $\left(T_{\mathrm{hoz}}\right)$ and the related horizontal propulsors that are useful, and not the dormant vertical propulsors, when the VTOL airplane is in forward flight mode. During a hover, the relation between the power $P$ of an electric motor and the generated thrust $T$ may be expressed as $P=c \cdot T^{1.5}[17]$, where $\mathrm{c}$ is a constant.

From Equation (3), mass $m$ and hence propulsor weight $W$ is proportional to power $P$ and therefore, we have

$$
W_{\text {penalty }}=\left(\frac{P_{\text {vert }}}{P_{\text {hoz }}}\right)=\left(\frac{T_{\text {vert }}}{T_{\text {hoz }}}\right)^{1.5}
$$

As mentioned, each propulsor of the ATRS- 45 will output a thrust of $0.3536 W_{\Sigma}$ and so adding the four thrusts together will give a total thrust magnitude of $1.4142 W_{\Sigma}$. As for the quadplane each vertical propulsor outputs a thrust, $T_{\text {vert }}$ of $0.25 W_{\Sigma}$. Since the quadplane has the same of total thrust of $1.4142 W_{\Sigma}$, then its forward horizontal thrust will be $T_{\text {hoz }}=1.4142 W_{\Sigma}-(4 \times 0.25)=0.4142 W_{\Sigma}$. This gives a thrust ratio, $T_{\text {vert }} / T_{\text {hoz }}$ of $0.25 / 0.4142=0.6036$, or $T_{\text {vert }}=60.36 \% T_{\text {hoz }}$ for each vertical propulsor, as shown in parentheses in Figure 4(a). Using the same analysis, each vertical propulsor of the ATRS- 45 will have $T_{\text {vert }}=50 \% T_{\text {hoz }}$. From the plot in Figure 3, if the hovering pitch angle $\rho$ were to increase to $60^{\circ}$ i.e., 
ATRS-60, one would obtain a total thrust magnitude of only $1.3660 W_{\Sigma}$, which means it has an "excess" thrust of $1.4142 W_{\Sigma}-1.3660 W_{\Sigma}=0.0482 W_{\Sigma}$. In order to include ATRS-60 in the weight penalty comparison, the "excess" thrust of $0.0482 W_{\Sigma}$ will be assigned to its horizontal propulsors.

Consequently, each horizontal propulsor of ATRS-60 will have a maximum $T_{\text {hoz }}$ of $0.4571 W_{\Sigma}$ at full throttle. Based on Equation (5), the $W_{\text {penalty }}$ for each vertical propulsor of the quadplane was calculated to be 0.4689 or $46.89 \%$ (expressed as percentage). The overall $W_{\text {penalty }}$ for the quadplane's 4 vertical propulsors then becomes $4 \times 0.4689=1.8756$, or $187.56 \%$. The interpretation of such result is that the quadplane is carrying a weight that is nearly twice that of its horizontal propulsor which could have otherwise been allocated for "useful" purposes such as forward propulsion, payload or fuel load. The $W_{\text {penalty }}$ values for the quadplane, as well as for the ATRS with hovering pitch angles $\rho=45^{\circ}$ and $\rho=$ $60^{\circ}$ are summarized in Table 1 . The $W_{\text {penalty }}$ expressed as percentage for the ATRS-45 and ATRS-60 are 70.71\%, and 31.02\%, respectively. These results suggested that both ATRS were able to achieve VTOL via retarded harrier with significantly lower weight penalty, $W_{\text {penalty }}$.

The thrust-to-weight ratio $\left(T_{\mathrm{hoz}} / W_{\Sigma}\right)$ is another useful parameter and thus it was included in Table 1 as it relates to maximum airspeed achievable. Let's assume the quadplane and the airplane with tandem roto-stabilizers have similar drag and neglecting any additional drag effect due to the presence of the boom structures on the quadplane. The ATRS is expected to have a higher maximum airspeed in level flight with maximum continuous power $\left(V_{H}\right)$ given that it has a higher thrust-to-weight ratio than the quadplane. Using the relation $T_{\max } \propto V_{H}^{2}$, the $V_{H}$ of the ATRS-45 was estimated to be about $30 \%$ higher than that of the quadplane. The thrust-to-weight ratio of the ATRS-60 is 0.8660 and the corresponding $V_{H}$ is approximately $45 \%$ higher than that of the quadplane. In manned flight applications, this will translate to arriving at destination sooner than the quadplane of equal propulsor weights. Overall, the analysis suggested that achieving VTOL via the retarded harrier maneuver is an efficient approach and that it confers higher cruising speed and lower weight penalty than a quadplane for the same propulsor weights. Furthermore, in our opinion, unlike the quadplane requiring boom structures for the vertical propulsors, the ATRS has better esthetics and possibly less aerodynamic drag while cruising.

Table 1 . Weight penalty as a percentage of $W_{\text {hoz }}$ and thrust-to-weight ratio for the quadplane, and the ATRS hovering at pitch angles of $45^{\circ}$ and $60^{\circ}$.

\begin{tabular}{cccc}
\hline & Quadplane & ATRS-45 & ATRS-60 \\
\hline $\begin{array}{c}W_{\text {penalty }} \text { for each vertical propulsor } \\
\left(\% \text { of } W_{\text {hoz }}\right)\end{array}$ & 46.89 & 35.36 & 14.30 \\
$\begin{array}{c}W_{\text {penalty }} \text { for total vertical propulsors } \\
\left(\% \text { of } W_{\text {hoz }}\right)\end{array}$ & 187.56 & 70.71 & 28.60 \\
$\begin{array}{c}\text { Thrust-to-weight ratio } \\
\left(T_{\text {hoz }} / W_{\text {aircraft }}\right)\end{array}$ & 0.4142 & 0.7071 & 0.9142 \\
\hline
\end{tabular}


Despite the ATRS-60 having the lowest $W_{\text {penalty }}$ among the aircraft configurations considered, however such configuration in its as-it-is "self-sufficient" form has fundamental design constraints because it would require a generally short fuselage or a relatively long landing gears in order to perform retarded harrier at $\rho=60^{\circ}$ during takeoff. We hereby proposed the concept of "distributed VTOL system" (DVS). Traditionally, designs of VTOL airplanes were very much based on "self-sufficient" approach in which the components and systems required to achieve VTOL is built-in and forming an integral part of the aircraft. In the DVS approach, VTOL components and systems are partially external to the airplane. Figure 5 shows a DVS approach involving a $60^{\circ}$ inclination ramp structure that would enable the ATRS-60 to achieve VTOL. The airplane would climb up the ramp under its own power using its horizontal thrusts, $T_{\text {hoz }}$ as shown in Figure 5 (a). Once the pitch angle $\rho$ of the airplane has reached $60^{\circ}$, the vertical motors are energized. And when each horizontal propulsor generates a $T_{\text {hoz }}$ of $0.4330 W_{\Sigma}$ and each vertical propulsor generates a $T_{\text {vert }}$ of $0.25 W_{\Sigma}$, vertical takeoff is initiated as shown in Figure 5(b). The white arrow indicates the flight path vector of the ATRS- 60 - that is, the pitch angle, $\rho$ is $60^{\circ}$ and the flight path angle is $90^{\circ}$. During vertical landing, the reverse process occurs. In our previous work relating to typhoon application, the airplane made its way towards the docking surface using only the harrier maneuver during landing and that necessarily involved some forward airspeed prior to contact with the docking surface [18].

\subsection{Experimental Demonstration of Retarded Harrier Maneuver}

\subsubsection{Materials and Method}

A small mono-wing remotely piloted ATRS-45 as shown in Figure 6 was built to demonstrate the concept of retarded harrier maneuver and the airplane was designed to hover at $\rho=45^{\circ}$. The horizontal and vertical propulsors were powered by brushless electric motors (DYS Storm 2207, $2300 \mathrm{kV}$ ) and the airplane has a total wing span of $650 \mathrm{~mm}$ and a wing area of $12.52 \mathrm{dm}^{2}$. The $W_{\Sigma}$ of the airplane was approximately $630 \mathrm{~g}$. The fuselage was constructed using simple dual-beam square carbon tubes with cross-sectional dimensions of $5 \times 5 \mathrm{~mm}$ and length of $520 \mathrm{~mm}$. Airframe components, such as mounting plates for the motors and

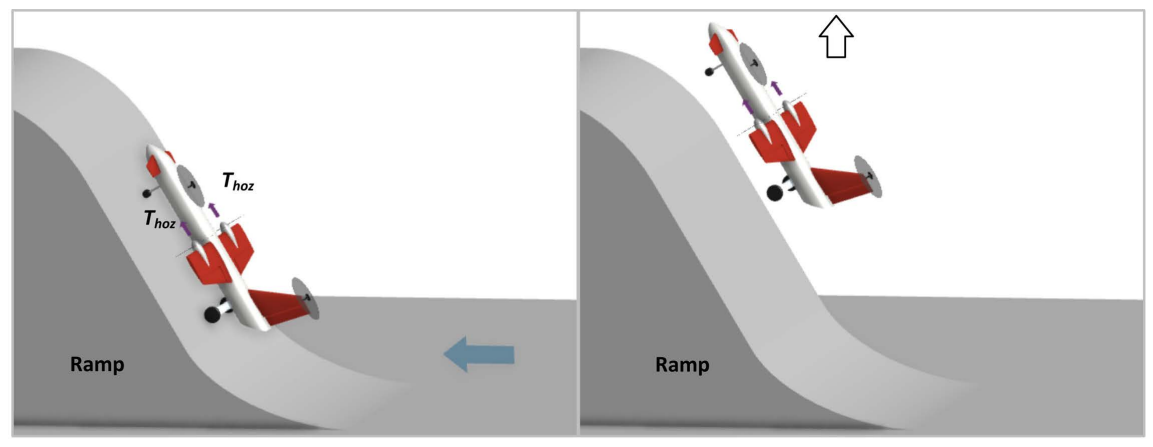

(a)

(b)

Figure 5. A concept of "distributed VTOL system" (DVS) that would enable the ATRS-60 to perform vertical takeoff without the need for long landing gears. 


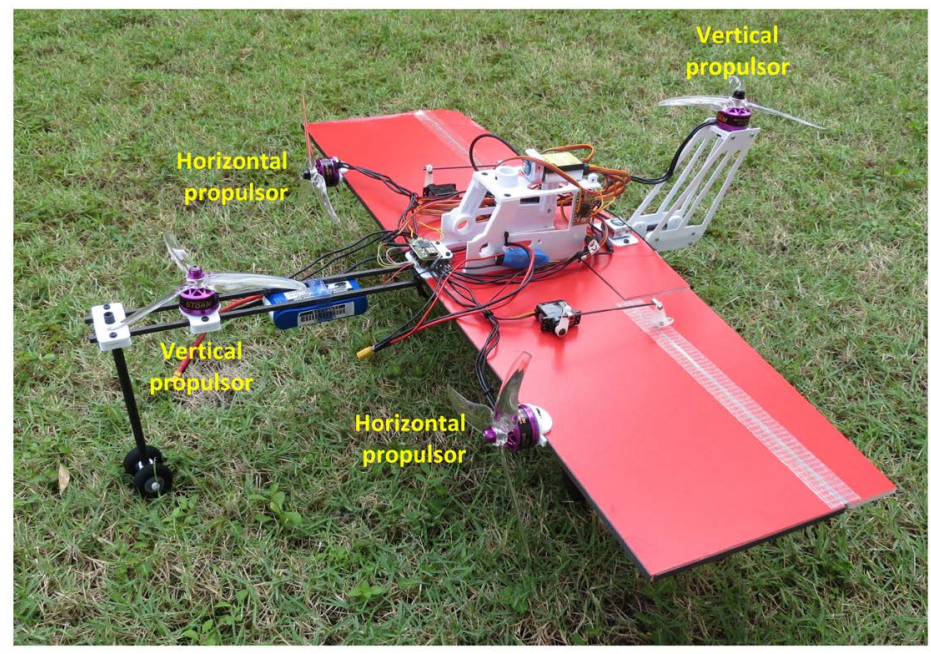

Figure 6. A small remotely piloted ATRS-45.

landing gears, were 3D-printed with polylactic acid (PLA) material. Each of the vertical propulsors was placed about $22 \mathrm{~cm}$ away from the center of gravity (C.G.) of the airplane and 3-bladed counter-rotating propellers (T-Motor T6143) were used. Identical propulsor pair was used for the horizontal propulsion and the motors were mounted near the leading edges of the wings. Differential thrust of the horizontal propulsors was used to actuate yaw. Ailerons with relatively large surface area immersed in the strong propeller wash generated by the horizontal propulsors ensured authoritative roll control during hyper-STOL and VTOL.

The airplane's flight controller consisted of two Teensy boards [19] and three commercially available single-axis gyros (Futaba GY 520, and MICROBEAST's yaw gyro [20]), all of which were programmed to operate in angular hold mode. We designed the gyro system so that gains for the roll, pitch and yaw could be easily adjusted and optimized while in-flight using 3 independent knobs on the radio transmitter. This made gyro gain tuning easier and without having to bring a laptop or tablet to the flying field.

\subsubsection{Vertical Flight}

Field testing revealed that the remotely piloted ATRS- 45 indeed hovered with a pitch angle, $\rho$ of $43^{\circ}\left( \pm 0.5^{\circ}\right)$, as shown in Figure 7. Both the horizontal and vertical propulsors have the same output thrust and are controlled by the throttle stick on the radio transmitter. The pitch angle, $\rho$ was determined from simple photographic method. An inclinometer with accuracy of $0.1^{\circ}$ was attached to the camera that captured the sideview of the airplane as it performed hovering in calm wind conditions (wind speed $<5 \mathrm{~km} \cdot \mathrm{hr}^{-1}$ ). The camera was held in level position when acquiring the images. The measured pitch angle, $\rho$ having a value of $2^{\circ}$ less than $45^{\circ}$ may be attributed to the presence of light headwind. The pitch and yaw controls were responsive, and so was the roll control which was handled by the relatively large ailerons. Overall, the vertical flights and hovering were stable, precise and predictable (see Video 1 for details). 


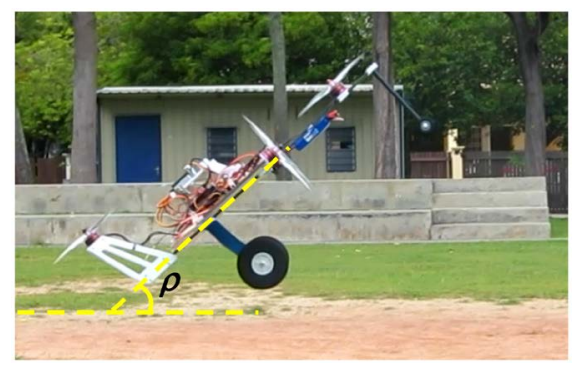

Figure 7. ATRS-45 in a hover.

\subsubsection{Horizontal Flight}

This section demonstrates the horizontal flight and flight transition characteristics of the ATRS-45. Elevators in the form of canard surfaces were added to the front of the aircraft and actuated by a servo mounted underneath the fore unit of the vertical propulsor (Figure 8). The total area of the canard surfaces was 5.2\% of that of the main wings. Figures $9(a)-(f)$ depict a hyper-STOL sequence performed with a target pitch angle, $\rho$ of about $40^{\circ}$ and in moderate breeze with slight turbulence. The required takeoff ground roll was well under 10 meters.

Once airborne, the pitch angle, $\rho$ was gradually reduced to enable the aircraft to enter near-horizontal flight mode. See Video 2 for the transition process. Figure 10(a) shows the airplane in forward flight with the vertical propulsors remained active. The flight controller was programmed so that the vertical propulsors could be de/activated with built-in linear ramp via a two-position switch on the radio transmitter whenever full transition to pure fixed-wing mode is needed. Deactivation of the tandem roto-stabilizers (around airspeed $50 \mathrm{~km} \cdot \mathrm{hr}^{-1}$ ) was found to result in flight transition that was smooth and seamless. Additionally, no change in the pitch gyro gain setting was required. The flight test also provided validation that the surface area of the canard was appropriate and having adequate authority to control the pitch of the airplane during forward flight. Figure 10(b) shows the fore unit of the vertical propulsor in deactivated state with the propeller and the logo "dys" on the motor clearly visible. Use of the canard surfaces for pitch control during cruising led to an energy efficient flight. It also facilitates conventional runway landing in the event of malfunctioning of the vertical propulsors. See Video 3 (FPV) for transition characteristics between vertical and horizontal flight modes. The video also shows gyro-stabilized canard surfaces in action.

To revert back to "vertical flight" mode necessary for hyper-short or vertical landing, the tandem vertical propulsors simply needed to be re-activated via the transmitter switch. Again, the transition was safe and seamless requiring no control intervention or corrective action from the remote pilot on the ground. Figure 11 shows the aircraft performing a near vertical landing using the retarded harrier maneuver (see Video 4 for details). The stronger the headwind, the lesser is the pitch angle needed to achieve a hover or vertical flight. In other words, the retarded harrier approach exhibits robustness against wind and enables an airplane to retain hyper-STOL or VTOL capability under windy conditions, 


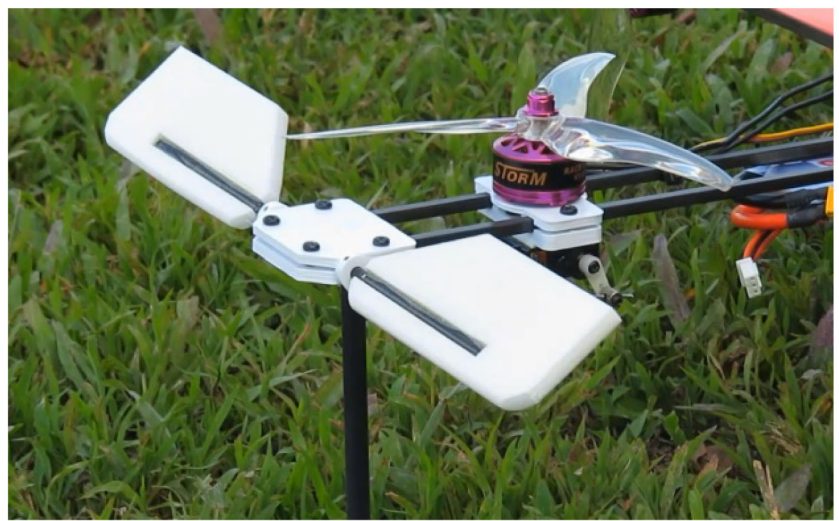

Figure 8. Addition of canard surfaces for pitch control during forward flight.

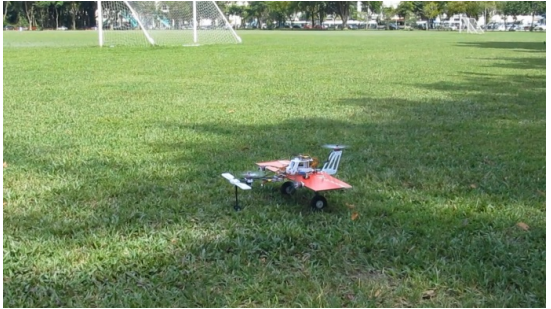

(a)

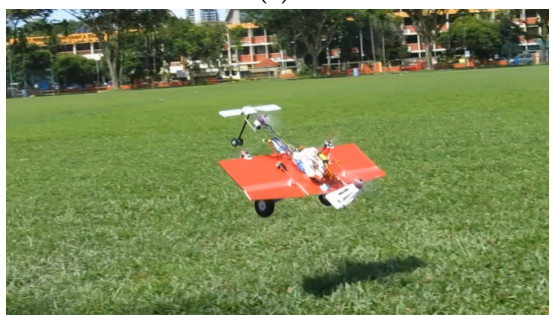

(c)

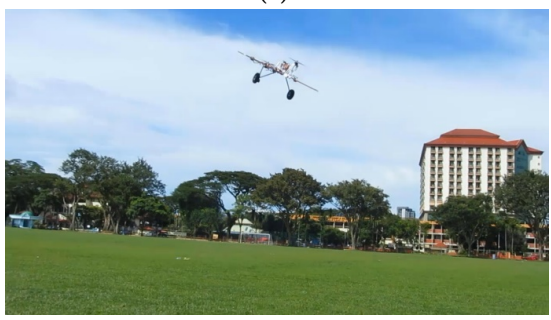

(e)

Figure 9. Hyper-STOL sequence.

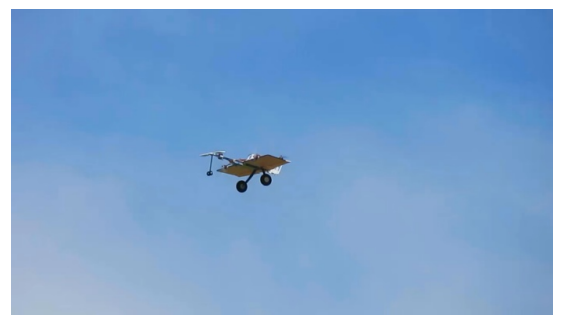

(a)

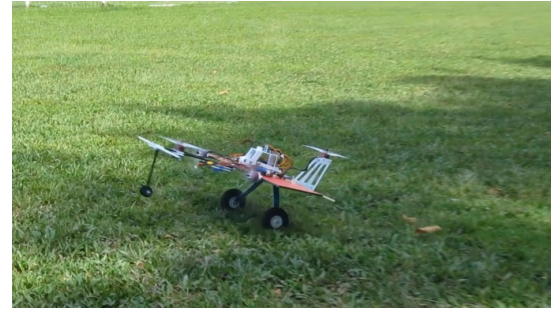

(b)

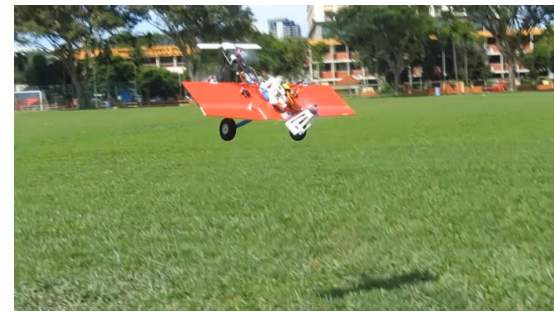

(d)

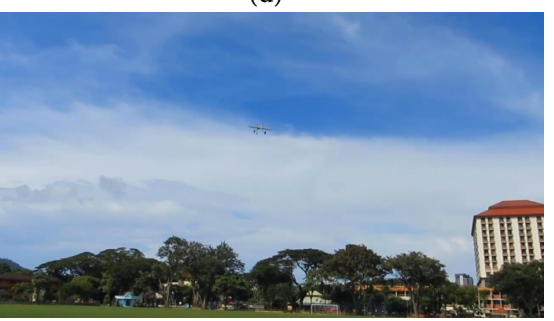

(f)

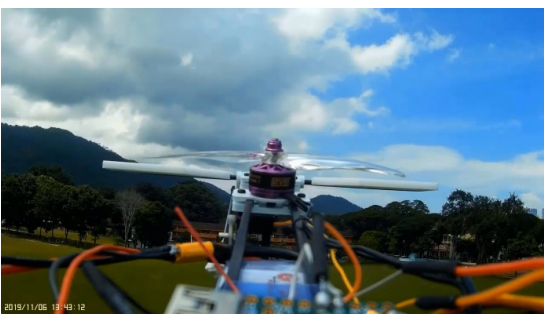

(b)

Figure 10. (a) Forward flight of the ATRS-45 with its vertical propulsors active, and (b) an onboard view showing the fore vertical propulsor deactivated. 


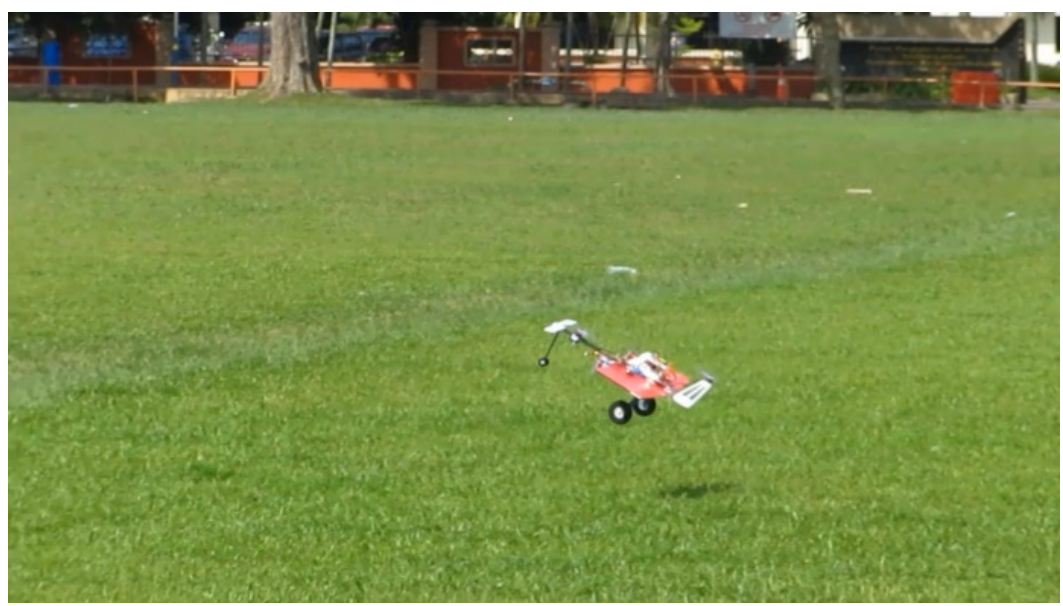

Figure 11. The ATRS-45 performing a near vertical landing using the retarded harrier maneuver.

such as carrying out first responder missions soon after the passage of a severe tropical cyclone where roads are damaged and runways are compromised. In fact, any VTOL airplane having basic quadplane configuration can perform the retarded harrier maneuver by simultaneously activating its horizontal and vertical propulsors and applying a positive pitch angle during takeoff and landing.

\section{Conclusion}

We had presented a new maneuver termed as retarded harrier and it is applicable to un/manned fixed-wing aircraft for achieving S/VTOL flight with an improved forward flight performance than a typical quadplane in terms of weight, speed and esthetics. During the retarded harrier maneuver, the airplane assumes a nose-up attitude with positive pitch angle so that the horizontal and vertical propulsors are active and contributing to lift via resolution of vectors. The aircraft achieved hovering when horizontal components canceled out. The concept of "distributed VTOL system" (DVS) was proposed to enable a VTOL airplane to achieve VTOL via retarded harrier without having to use long landing gears. A small remotely piloted aircraft with tandem roto-stabilizers was also presented to demonstrate retarded harrier maneuver. Phases of flight demonstrated included hovering, flight transitions and forward flight. The flight transition to forward flight and vice versa was practically seamless even in the presence of headwind and this is attributed to the unique attitude in which the aircraft assumed while performing the retarded harrier. Any fixed-wing aircraft having basic quadplane configuration can adequately perform VTOL via the retarded harrier maneuver by simultaneously activating its horizontal and vertical propulsors and applying a positive pitch angle.

\section{Conflicts of Interest}

The authors declare no conflicts of interest regarding the publication of this paper. 


\section{References}

[1] Wikipedia (2021) Tiltrotor. https://en.wikipedia.org/wiki/Tiltrotor\#History

[2] The Modelling News (2020) Amusing Hobby's New 48th Scale Kit in the Test Shot Phase-The WWII German VTOL Weserflug P.1003/1.

https://www.themodellingnews.com/2020/08/amusing-hobbys-new-48th-scale-kit-i $\underline{\text { n.html }}$

[3] Wikipedia (2020) Tiltwing. https://en.wikipedia.org/wiki/Tiltwing

[4] Vertical Flight Society (2019) History of VTOL Aircraft Design-Tilt Wings \& Tilt Rotors. https://www.youtube.com/watch?v=0JK5dS_StGM

[5] ArduPilot Dev Team (2019) QuadPlane Overview. https://ardupilot.org/plane/docs/quadplane-overview.html

[6] Vertical Flight Society (2021) Uber Elevate eCRM-003. https://evtol.news/uber-elevate-ecrm-003/

[7] Vertical Flight Society (2021) Aurora Flight Sciences Pegasus PAV. https://evtol.news/aurora/

[8] Vertical Flight Society (2021) EmbraerX Eve. https://evtol.news/embraer/

[9] Selig, M.S. (2010) Modeling Full-Envelope Aerodynamics of Small UAVs in Realtime. Proceedings of the AIAA Atmospheric Flight Mechanics Conference, Ontario, 2-5 August 2010, 1-35. https://doi.org/10.2514/6.2010-7635

[10] Selig, M.S. (2014) Real-Time Flight Simulation of Highly Maneuverable Unmanned Aerial Vehicles. Journal of Aircraft, 51, 1705-1725.

https://doi.org/10.2514/1.C032370

[11] Fédération Aéronautique Internationale (2021) Powered Aerobatics. https://www.fai.org/page/civa-powered-aerobatics

[12] Red Bull (2013) Airplane vs. Parachutist-Martin Sonka \& Petr Mestak 2013. https://www.youtube.com/watch?v=S7WqwNiyP3k

[13] Wikipedia (2020) 3D Aerobatics. https://en.wikipedia.org/wiki/3D_Aerobatics

[14] Poh, C.-H. and Poh, C.-K. (2014) Radio Controlled “3D Aerobatic Airplanes" as Basis for Fixed-Wing UAVs with VTOL Capability. Open Journal of Applied Sciences, 4, 515-521. http://doi.org/10.4236/ojapps.2014.412050

[15] Poh, C.-K. and Poh, C.-H. (2018) Roto-Stabilizer for Superb Pitch-Related Post-Stall Maneuvers and STOL. Advances in Aerospace Science and Technology, 3, 61-70. https://doi.org/10.4236/aast.2018.33005

[16] Poh, C.-K. and Poh, C.-H. (2020) Roto-Stabilizer: Experimental Demonstration of Hyper-STOL and Vertical Flight. Aerospace Europe Conference (AEC 2020), Bordeaux, 25-28 February 2020, 199.

[17] Rotaru, C. and Todorov, M. (2018) Helicopter Flight Physics. IntechOpen, London, 19-47. https://doi.org/10.5772/intechopen.71516

[18] Poh, C.-K. and Poh, C.-H. (2016) Journey to the Typhoon. Advances in Aerospace Science and Technology, 1, 21-31. https://doi.org/10.4236/aast.2016.11003

[19] PJRC (2021) Teensy 2.0 Development Board. https://www.pjrc.com/store/teensy.html

[20] Beastx (2019) Microbeast (Version 3). http://www.beastx.com/eng/microbeast.php 